\title{
DROMO PROPAGATOR REVISITED
}

\author{
H. Urrutxuał, M. Sanjurjo-Rivo ${ }^{\dagger}$ and J. Peláez ${ }^{\dagger}$
}

\begin{abstract}
In year 2000 a house-made orbital propagator was developed by the SDG-UPM (former Grupo de Dinámica de Tethers) based in a set of redundant variables including Euler parameters. This propagator was called $\mathrm{DROMO}^{*}$ and it was mainly used in numerical simulations of electrodynamic tethers. It was presented for the first time in the international meeting $V$ Jornadas de Trabajo en Mecánica Celeste, held in Albarracín, Spain, in 2002 (see reference 1). The special perturbation method associated with DROMO can be consulted in the paper. $^{2}$ In year 1975 , Andre Deprit in reference 3 proposes a propagation scheme very similar to the one in which DROMO is based, by using the ideal frame concept of Hansen. The different approaches used in references 3 and 2 gave rise to a small controversy. In this paper we carried out a different deduction of the DROMO propagator, underlining its close relation with the Hansen ideal frame concept, and also the similarities and the differences with the theory carried out by Deprit in 3. Simultaneously we introduce some improvements in the formulation that leads to a more synthetic propagator.
\end{abstract}

\section{INTRODUCTION}

In 1975 Deprit in ${ }^{3}$ proposed a new set of elements to describe Keplerian motions subject to perturbing forces. That work was followed by other papers (see references ${ }^{4-7}$ ) that involved the use of Euler parameters (quaternions) in the propagation of perturbed Keplerian orbits. Quaternions had been widely used in the analysis of the attitude dynamics of a rigid body (see for example 8), but they had not been commonly used in orbital mechanics. This approach was proposed for the first time, as far as the authors knowledge, in. ${ }^{3}$

Some other papers presented similar formulations (see 9-12) with a common characteristic: the use of Euler parameters forming part of a set of redundant variables. Thus the propagation of a perturbed orbit became a problem of order seven, or higher, instead of six as in the classical formulation of that problem (Cowell or Encke's methods). Rising the order of the system is not a big burden in computer algorithms (see 13) and, in some cases, leads to substantial improvements of performance.

In year 2000 a house-made orbital propagator was developed by the SDG-UPM (former Grupo de Dinámica de Tethers). The aim of the project was to develop a regular, robust and efficient propagator. Regular means that the propagator is free of singularities. Robust means that it should be numerically stable. Efficient means that the propagator should render accurate results with low time-consumption and share a common formulation for elliptical, parabolic and hyperbolic problems. How accurate the results are and how quick the propagator provide them constitute a metric of its performance.

To fulfill these requirements, a special perturbation method of variation of parameters (VOP) based in a set of redundant variables including quaternions was considered. That propagator was called DROMO and initially it was mainly used in numerical simulations of electrodynamic tethers.

Special perturbation methods are characterized by some flexibility to deal with different perturbation forces in an easy manner. The VOP method, in particular, presents some advantages: a lesser accumulation of errors, fewer integration steps and a deeper insight in the physics of the problem. ${ }^{3}$ Some of these advantages have

\footnotetext{
†Space Dynamics Group (SDG-UPM), ETSI Aeronáuticos, Technical University of Madrid (UPM), Pz Cardenal Cisneros 3, Madrid 28040, Spain, (e-mail: sdg.aeroespacial@upm.es)

*The word DROMO is derived from the old Greek word $\delta \rho \delta$ ó that means, approximately, rushing and it appears as suffix in many words like velodrome, hippodrome, etc.
} 
been underlined in recent works as in. ${ }^{14}$ Although, and according to Danby, ${ }^{15}$ VOP methods may suffer from problems with function evaluations depending on the perturbing force.

The fundamentals of the VOP method are unique; however, the motion can be described by a number of different sets of elements. $\operatorname{In}^{16}$ a collection of different sets can be found. Previous works show the possibility to use even non-osculating elements in the description of the perturbed two body problem: ${ }^{17}$ the possibility of choosing the "gauge" can be useful in some cases. Nevertheless, in a general purpose propagator the parameters should not lack physical meaning. Therefore, a selection of the suitable set of elements for the Keplerian motion must be made. According to the philosophy of design of DROMO, the parameters should keep the general advantages of the VOP method and also avoid singularities in the description of the motion.

In DROMO, the parameters were selected with a dynamical and geometrical criteria and according to the following approach. The description of the motion is broken up into three steps: movement of a reference frame attached to the orbital plane, osculating trajectory in the orbital plane and pin-point the position in the osculating trajectory as a function of time.

This approach is closely related to the ideal frame concept introduced by Hansen in the XIX century. The Hansen's ideal frame is attached to the orbital plane and allows us to separate the periodic perturbations in the orbital plane from those of the orbital plane itself. The other important and related feature of the ideal frame is that the equations of relative and absolute motions have the same form, because the fictitious forces of Coriolis and centrifugal are equal and opposed. The components of a quaternion describing the attitude of the ideal frame are used in DROMO as parameters.

The special perturbation method associated with DROMO was presented for the first time in the international meeting V Jornadas de Trabajo en Mecánica Celeste, held in Albarracín, Spain, in 2002 (see 1). A more detailed presentation of DROMO took place in the 15th AAS/AIAA Spaceflight Mechanics Meeting held in Copper Mountain, CO, in 2005 (see 18) and, finally, in the paper. ${ }^{2}$

The DROMO propagator - in the version just described in ref. 2- consists of a set of eight ODE's whose independent variable is a fictitious time provided by a generalized Sundman transformation which reduces to the true anomaly when the motion is purely Keplerian. The DROMO set of dependent variables is redundant and seven dependent variable are generalized elements, that is, they take constant values in the Keplerian motion. This characteristic distinguishes DROMO from other similar schemes like for example ${ }^{9-12}$ and. ${ }^{19}$ $\mathrm{In}^{19}$ authors carried out a propagator with also eight ODE's; however, the dependent variables used in ${ }^{19}$ are not generalized elements.

Andre Deprit in, 3,4 and taking the ideal frame concept of Hansen as starting point, proposed a propagation scheme which is almost equal to the scheme in which DROMO is based. The different approaches used in ${ }^{3}$ and $^{2}$ gave rise to a small controversy. In this paper we carried out a different deduction of the DROMO propagator, underlining its close relation with the Hansen ideal frame concept, and also the similarities and the differences with the theory carried out by Deprit in. ${ }^{3}$ Simultaneously we introduce some improvements in the formulation that leads to a more synthetic propagator.

\section{BASIC ELEMENTS OF DROMO'S THEORY}

Let $m$ be the mass of a particle $M$ moving in a fixed inertial frame $O x_{1} y_{1} z_{1}$ (solid 1). The origin $O$ is the center of mass of a celestial body. Therefore, $M$ is acted upon by: 1) the gravitation of that body which is the main controlling force and 2) the remaining forces which are included in the perturbing force $\boldsymbol{F}_{p}$. Under these conditions, $M$ is moving governed by the equation:

$$
m \frac{\mathrm{d}^{2} \boldsymbol{x}}{\mathrm{d} t^{2}}=-\frac{m \mu}{|\boldsymbol{x}|^{3}} \boldsymbol{x}+\boldsymbol{F}_{p}
$$

which should be integrated from appropriated initial conditions:

$$
\text { at } \quad t=t_{0}: \quad \boldsymbol{x}=\boldsymbol{x}_{0}, \quad \boldsymbol{v}=\boldsymbol{v}_{0}
$$




\section{Orbital frame}

Let $M x y z$ be the orbital frame (see figure 1) defined as follows: 1) the axis $M x$ along the radius vector $\boldsymbol{x}$ of $M, 2$ ) the plane $M x y$ defined by the position vector $\boldsymbol{x}$, the velocity vector $\dot{\boldsymbol{x}}=\boldsymbol{v}$ and oriented in such a way that $\boldsymbol{v} \cdot \boldsymbol{j} \geq 0$, and 3) the axis $M z$ completes a righthanded orthogonal frame. The plane $M x y$ is the orbital plane. In the pure Keplerian motion $\left(\boldsymbol{F}_{p}=\mathbf{0}\right)$, it is fixed to the inertial frame $O x_{1} y_{1} z_{1}$ and it contains the trajectory of $M$. In the perturbed motion $\left(\boldsymbol{F}_{p} \neq \mathbf{0}\right)$ the orbital plane is moving into the inertial frame but it contains the osculating orbit.

In reference 2 the orbital frame $M x y z$ plays a central role. However it is more convenient to use the frame $O x y z$ (see figure 1) with origin in $O$. The planes $O x y$ and $M x y$ coincide from a geometrical point of view. However, they are different planes from a kinematical point of view. Which one is the real orbital plane? Later on we will give an answer.

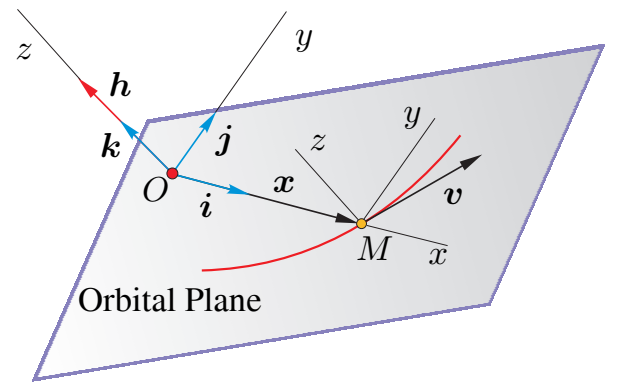

Figure 1. Orbital frame

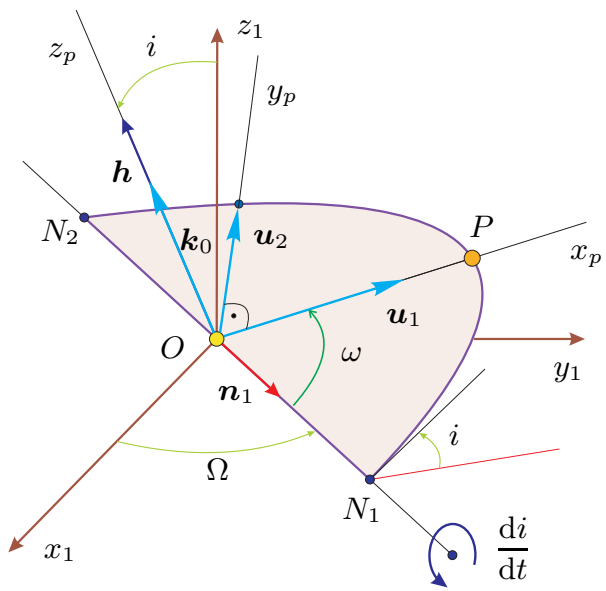

Figure 2. Perifocal frame

\section{Situation of the orbital plane. Reference frames}

In the pure Keplerian motion the orbital plane is situated by three Euler angles that take constant values: the longitude of the ascending node $\Omega$, the inclination $i$ and the argument of pericenter $\omega$. Usually, these angles are associated with the frame $O x_{0} y_{0} z_{0}$ or the perifocal frame $O x_{p} y_{p} z_{p}$ at the initial instant. Figure 2 shows an sketch of the perifocal frame $O x_{p} y_{p} z_{p}$.

Alternatively, the perifocal frame $O x_{p} y_{p} z_{p}$ can be situated by means of a unit quaternion $\mathbf{n}$ whose coordinates are:

$$
\mathbf{n}=\eta_{4}+\left(\eta_{1}, \eta_{2}, \eta_{3}\right)
$$

The unit vectors $\left(\boldsymbol{u}_{1}, \boldsymbol{u}_{2}, \boldsymbol{k}_{0}\right)$ of the perifocal frame $O x_{p} y_{p} z_{p}$ in terms of the unit vectors of the inertial frame are given by:

$$
\left\lfloor\boldsymbol{u}_{1}, \boldsymbol{u}_{2}, \boldsymbol{k}_{0}\right\rceil=\left\lfloor\boldsymbol{i}_{1}, \boldsymbol{j}_{1}, \boldsymbol{k}_{1}\right\rceil P_{0}
$$

where the matrix $P_{0}$, in terms of the quaternion $\mathbf{n}$, is given by:

$$
P_{0}=\left(\begin{array}{ccc}
1-2\left(\eta_{2}^{2}+\eta_{3}^{2}\right) & 2 \eta_{1} \eta_{2}-2 \eta_{4} \eta_{3} & 2 \eta_{1} \eta_{3}+2 \eta_{4} \eta_{2} \\
2 \eta_{1} \eta_{2}+2 \eta_{4} \eta_{3} & 1-2\left(\eta_{1}^{2}+\eta_{3}^{2}\right) & 2 \eta_{2} \eta_{3}-2 \eta_{4} \eta_{1} \\
2 \eta_{1} \eta_{3}-2 \eta_{4} \eta_{2} & 2 \eta_{3} \eta_{2}+2 \eta_{4} \eta_{1} & 1-2\left(\eta_{1}^{2}+\eta_{2}^{2}\right)
\end{array}\right)
$$

In terms of the classical elements $P_{0}$ is given by:

$$
P_{0}=\left(\begin{array}{ccc}
+\cos \Omega \cos \omega-\cos i \sin \Omega \sin \omega & -\cos \Omega \sin \omega-\cos i \sin \Omega \cos \omega & +\sin i \sin \Omega \\
+\sin \Omega \cos \omega+\cos i \cos \Omega \sin \omega & -\sin \Omega \sin \omega+\cos i \cos \Omega \cos \omega & -\sin i \cos \Omega \\
+\sin i \sin \omega & +\sin i \cos \omega & \cos i
\end{array}\right)
$$


We can move from a representation of matrix $P_{0}$ to the other one taking into account the relations:

$$
\begin{aligned}
\eta_{1} & =\sin \left(\frac{i}{2}\right) \cos \left(\frac{\Omega-\omega}{2}\right) & \eta_{2} & =\sin \left(\frac{i}{2}\right) \sin \left(\frac{\Omega-\omega}{2}\right) \\
\eta_{3} & =\cos \left(\frac{i}{2}\right) \sin \left(\frac{\Omega+\omega}{2}\right) & \eta_{4} & =\cos \left(\frac{i}{2}\right) \cos \left(\frac{\Omega+\omega}{2}\right)
\end{aligned}
$$

Finally, note that in order to situate the frame $O x_{0} y_{0} z_{0}$ at the initial time another additional parameter is required: the value of the initial true anomaly $\theta_{0}$.

\section{KINEMATICS ASPECTS OF DROMO'S THEORY}

First of all we should clarify the orbital plane concept. In the perturbed motion of $M$ the orbital plane is no longer fixed to the inertial frame.

Let us consider $N$ sheets of paper, contained in the plane $O x y$, which are in motion relative to each other. From a geometric point of view all of them define the same plane. However, from a kinematical point of view things are different because each sheet of paper is a different rigid body. Let us attache a frame $O x_{i} y_{i} z_{i}$, $i=1, \ldots, N$ to each one of the sheets (with the same origin $O$ in order to avoid irrelevant details). In such a case the relative motion between the sheets is a rotation around the $O z$ axis since all the frames $O x_{i} y_{i} z_{i}$, $i=1, \ldots, N$ share this axis and the same unit vector: $\boldsymbol{k}_{i} \equiv \boldsymbol{k}$.

If $\boldsymbol{\omega}_{i}$ is the inertial angular velocity of the frame $O x_{i} y_{i} z_{i}, i=1, \ldots, N$ we have:

$$
\frac{\mathrm{d} \boldsymbol{k}}{\mathrm{d} t}=\boldsymbol{\omega}_{i} \times \boldsymbol{k}, \quad \forall i=1, \ldots, N
$$

and the cross product with $k$ provides:

$$
\boldsymbol{\omega}_{i}=\underbrace{\boldsymbol{k} \times \dot{\boldsymbol{k}}}_{\| \text {to the plane } O x y}+\underbrace{\boldsymbol{k}\left(\boldsymbol{\omega}_{i} \cdot \boldsymbol{k}\right)}_{\perp \text { to the plane } O x y}
$$

Therefore, the angular rates $\boldsymbol{\omega}_{i}, i=1, \ldots, N$ share the same component parallel to the plane Oxy; their normal components are different each others.

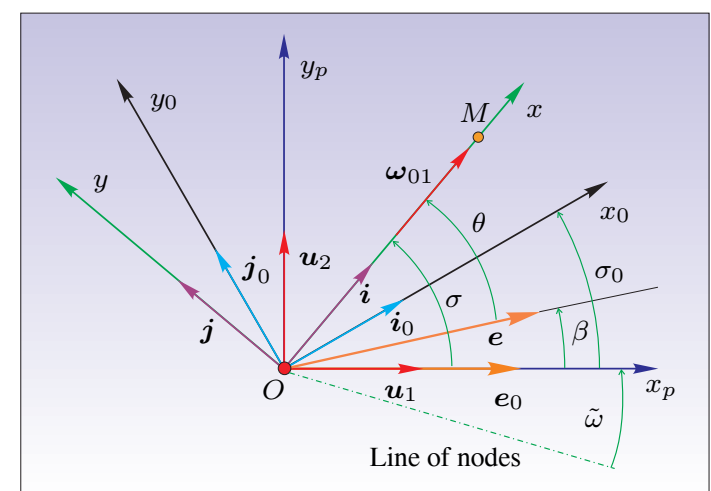

Figure 3. Kinematics elements of DROMO

\section{Ideal frame concept}

The question: which one of the sheets is really the orbital plane? Our answer: the one whose angular velocity lies in the plane $O x y$. In this paper the orbital plane defined in this way will be denoted as solid 0 . Many different frames can be attached to the orbital plane; they have been called ideal frames by Hansen as remember Deprit in 3,4. Two of these ideal frames should be underlined.

The first one is defined by the initial conditions $\left(\boldsymbol{x}_{0}, \boldsymbol{v}_{0}\right)$. We denote it by $O x_{0} y_{0} z_{0}$ and it is called the departure frame. The second one coincides with the perifocal frame at the initial time. We denoted it by $O x_{p} y_{p} z_{p}$ and it is called the departure perifocal frame. Both frames move in the inertial space and share the same angular velocity:

$$
\boldsymbol{\omega}_{01}=\boldsymbol{k} \times \dot{\boldsymbol{k}}
$$

which is the angular velocity of the solid 0 . As we show later on, it depends on the perturbation force $\boldsymbol{F}_{p}$. From now on, the orbital plane will be, in this paper, the solid 0 which can be defined by anyone of the specified ideal frames. Figure 3 shows both ideal frames and also the orbital frame. 


\section{Composition of motions and some kinematic elements}

Let us number the particle $M$ as body 2. The underlying theory of DROMO can be well understood if the inertial motion is separated into: 1) the motion of the particle $M$ relative to the orbital plane and 2) the motion of the orbital plane relative to the inertial space.

Two main rigid bodies are involved, namely: the inertial frame (solid number 1) and the orbital plane (solid number 0). In this paper the orbital plane is attached to the departure perifocal frame $O x_{p} y_{p} z_{p}$.

The theory of motion's composition provides:

$$
\boldsymbol{v}_{21}^{M}=\boldsymbol{v}_{20}^{M}+\boldsymbol{v}_{01}^{M}
$$

where $\boldsymbol{v}_{21}^{M}$ is the inertial velocity of $M$ and $\boldsymbol{v}_{20}^{M}$ its velocity relative to the orbital plane. Note that $\boldsymbol{v}_{01}^{M}$ is the inertial velocity of the point $M$ of the solid 0 , that is, the point of that body which coincides with $M$.

The inertial motion of the orbital plane is a pure rotation about the origin $O$, which keeps a fixed position. The angular velocity $\boldsymbol{\omega}_{01}$ describes completely that motion. Shortly we will show that this angular velocity $\boldsymbol{\omega}_{01}$ lies along the $O x \equiv M x$ axis of the orbital frame, i.e., it is $\boldsymbol{\omega}_{01}=\omega_{01} \boldsymbol{i}$ and its intensity $\omega_{01}$ depends on the perturbation force $\boldsymbol{F}_{p}$ (see relation (15) in page 1814). Therefore the instantaneous rotation axis of the motion of the orbital plane is the $O x$ axis. As a consequence the velocity of the particle $M$ vanishes: $\boldsymbol{v}_{01}^{M}=\mathbf{0}$. Thus the absolute and relative velocities of $M$ are the same: $\boldsymbol{v}_{21}^{M}=\boldsymbol{v}_{20}^{M}$. This important result drove Hansen to introduce the ideal frame concept.

Let $\sigma$ be the angle between the $O x$ axis and the $O x_{p}$ axis. Figure 3 shows that angle and the true anomaly $\theta$ of $M$ in the osculating orbit. At the initial time $t_{0}$ both angles are equal

$$
\text { at } \quad t=t_{0}: \quad \sigma_{0}=\theta_{0}
$$

and they are determined by the initial conditions $\left(\boldsymbol{x}_{0}, \boldsymbol{v}_{0}\right)$. But later on they are different due to the perturbation $\boldsymbol{F}_{p}$.

Note that the orbital frame $O x y z$ is not attached to the orbital plane; in fact, it is a new rigid body (solid number 3). The motion of this frame relative to the orbital frame is a pure rotation about the $O z$ axis; the angular velocity and acceleration are: $\boldsymbol{\omega}_{30}=\dot{\sigma} \boldsymbol{k}, \boldsymbol{\alpha}_{30}=\ddot{\sigma} \boldsymbol{k}$. The angular velocity of $O x y z$ relative to the inertial frame is:

$$
\boldsymbol{\omega}_{31}=\boldsymbol{\omega}_{30}+\boldsymbol{\omega}_{01}=\dot{\sigma} \boldsymbol{k}+\omega_{01} \boldsymbol{i}
$$

\section{Non-dimensional variables}

Table 1 shows the characteristics values used to introduce non-dimensional variables. In the original version of DROMO we used the following values:

$$
L_{c}=\left|\boldsymbol{x}_{0}\right|, \quad \omega_{c}=\sqrt{\frac{\mu}{L_{c}^{3}}}
$$

Table 1. Characteristics Values

\begin{tabular}{|lc|lc|}
\hline Length & $L_{c}$ & times & $1 / \omega_{c}$ \\
Velocities & $\omega_{c} L_{c}$ & Accelerations & $\omega_{c}^{2} L_{c}$ \\
Ang. momentum & $\omega_{c} L_{c}^{2}$ & Forces & $m \omega_{c}^{2} L_{c}$ \\
\hline
\end{tabular}

and the non-dimensional time $\tau=\omega_{c}\left(t-t_{0}\right)$.

From now on we will use non-dimensional variables unless otherwise specified. For example, $(\boldsymbol{x}, \boldsymbol{v})$ will be non-dimensional vectors that correspond to the position and velocity of $M$ relative to the inertial space.

The equations that govern the motion takes the form:

$$
\frac{\mathrm{d}^{2} \boldsymbol{x}}{\mathrm{d} \tau^{2}}=-\frac{1}{r^{3}} \boldsymbol{x}+\boldsymbol{a}_{p}(\boldsymbol{x}, \boldsymbol{v}, \tau)
$$

where $r=|\boldsymbol{x}|$ and $\boldsymbol{a}_{p}=\frac{\boldsymbol{F}_{p}}{m L_{c} \omega_{c}^{2}}$. The vector $\boldsymbol{a}_{p}$ is the perturbing acceleration acting on $M$ and it is a function, in general, of its dynamical state: $(\tau ; \boldsymbol{x}, \boldsymbol{v})$. 


\section{Angular velocity of the orbital plane}

Let $\boldsymbol{h}=\boldsymbol{x} \times \boldsymbol{v}$ be the angular momentum of $M$. The unit vectors $(\boldsymbol{i}, \boldsymbol{j}, \boldsymbol{k})$ of the orbital frame $O x y z$ are:

$$
\boldsymbol{i}=\frac{\boldsymbol{x}}{|\boldsymbol{x}|}=\frac{\boldsymbol{x}}{r}, \quad \boldsymbol{k}=\frac{\boldsymbol{x} \times \boldsymbol{v}}{|\boldsymbol{x} \times \boldsymbol{v}|}=\frac{\boldsymbol{h}}{h}, \quad \boldsymbol{j}=\boldsymbol{k} \times \boldsymbol{i}
$$

The time derivative of vectors $(\boldsymbol{i}, \boldsymbol{k})$ are:

$$
\frac{\mathrm{d} \boldsymbol{i}}{\mathrm{d} \tau}=\frac{1}{r}\left[\boldsymbol{v}-\frac{\mathrm{d} r}{\mathrm{~d} \tau} \boldsymbol{i}\right], \quad \frac{\mathrm{d} \boldsymbol{k}}{\mathrm{d} \tau}=\frac{1}{h}\left[\frac{\mathrm{d} \boldsymbol{h}}{\mathrm{d} \tau}-\frac{\mathrm{d} h}{\mathrm{~d} \tau} \boldsymbol{k}\right]
$$

Let $\left(\omega_{1}, \omega_{2}, \omega_{3}\right)$ be the components of $\boldsymbol{\omega}_{31}$ in the orbital frame: $\boldsymbol{\omega}_{31}=\omega_{1} \boldsymbol{i}+\omega_{2} \boldsymbol{j}+\omega_{3} \boldsymbol{k}$. The time derivative of unit vectors $(\boldsymbol{i}, \boldsymbol{k})$ fulfill the relations

$$
\frac{\mathrm{d} \boldsymbol{i}}{\mathrm{d} \tau}=\boldsymbol{\omega}_{31} \times \boldsymbol{i}=\omega_{3} \boldsymbol{j}-\omega_{2} \boldsymbol{k}, \quad \frac{\mathrm{d} \boldsymbol{k}}{\mathrm{d} \tau}=\boldsymbol{\omega}_{31} \times \boldsymbol{k}=\omega_{2} \boldsymbol{i}-\omega_{1} \boldsymbol{j}
$$

As a consequence:

$$
\frac{1}{r}\left[\boldsymbol{v}-\frac{\mathrm{d} r}{\mathrm{~d} \tau} \boldsymbol{i}\right]=\omega_{3} \boldsymbol{j}-\omega_{2} \boldsymbol{k} \Rightarrow\left\{\begin{array}{l}
\omega_{3}=\frac{1}{r}(\boldsymbol{v} \cdot \boldsymbol{j}) \\
\omega_{2}=0
\end{array}\right\}, \quad \frac{1}{h}\left[\frac{\mathrm{d} \boldsymbol{h}}{\mathrm{d} \tau}-\frac{\mathrm{d} h}{\mathrm{~d} \tau} \boldsymbol{k}\right]=\omega_{2} \boldsymbol{i}-\omega_{1} \boldsymbol{j} \Rightarrow\left\{\begin{array}{l}
\omega_{2}=0 \\
\omega_{1}=-\frac{1}{h}\left(\boldsymbol{j} \cdot \frac{\mathrm{d} \boldsymbol{h}}{\mathrm{d} \tau}\right)
\end{array}\right\}
$$

Since $\boldsymbol{h}$ is governed by equation

$$
\frac{\mathrm{d} \boldsymbol{h}}{\mathrm{d} \tau}=\boldsymbol{x} \times \boldsymbol{a}_{p}
$$

the components of $\boldsymbol{\omega}_{31}$ turns out to be:

$$
\begin{aligned}
& \omega_{1}=-\frac{1}{h}\left(\boldsymbol{j} \cdot \frac{\mathrm{d} \boldsymbol{h}}{\mathrm{d} \tau}\right)=-\frac{1}{h} \boldsymbol{j} \cdot\left(\boldsymbol{x} \times \boldsymbol{a}_{p}\right)=\frac{r}{h}\left(\boldsymbol{a}_{p} \cdot \boldsymbol{k}\right) \\
& \omega_{3}=\frac{1}{r}(\boldsymbol{v} \cdot \boldsymbol{j})=-\frac{1}{r} \boldsymbol{v} \cdot(\boldsymbol{i} \times \boldsymbol{k})=\frac{1}{r^{2}}(\boldsymbol{k} \cdot \boldsymbol{h})=\frac{h}{r^{2}}
\end{aligned}
$$

In summary, the time derivative of the unit vectors are:

$$
\begin{array}{ll}
\frac{\mathrm{d} \boldsymbol{i}}{\mathrm{d} \tau}=+\omega_{3} \boldsymbol{j}, & \omega_{3}=\frac{h}{r^{2}} \\
\frac{\mathrm{d} \boldsymbol{j}}{\mathrm{d} \tau}=-\omega_{3} \boldsymbol{i}+\omega_{1} \boldsymbol{k}, & \\
\frac{\mathrm{d} \boldsymbol{k}}{\mathrm{d} \tau}=-\omega_{1} \boldsymbol{j}, & \omega_{1}=\frac{r}{h}\left(\boldsymbol{a}_{p} \cdot \boldsymbol{k}\right)
\end{array}
$$

From the expression $\boldsymbol{\omega}_{31}=\boldsymbol{\omega}_{30}+\boldsymbol{\omega}_{01}=\dot{\sigma} \boldsymbol{k}+\omega_{01} \boldsymbol{i}=\omega_{1} \boldsymbol{i}+\omega_{3} \boldsymbol{k}$ the following relations can be deduced:

$$
\boldsymbol{\omega}_{01}=\omega_{1} \boldsymbol{i}=\frac{r}{h}\left(\boldsymbol{a}_{p} \cdot \boldsymbol{k}\right) \boldsymbol{i}, \quad \frac{\mathrm{d} \sigma}{\mathrm{d} \tau}=\frac{h}{r^{2}}
$$

The first one shows that the angular velocity of the orbital plane $\boldsymbol{\omega}_{01}$ is contained on the $O x$ axis, as we stated previously, and only depends on the component of the perturbing acceleration which is normal to the orbital plane. The second one gives the time evolution of the angle $\sigma$.

\section{DYNAMICS ASPECTS OF DROMO'S THEORY}

In DROMO the inertial motion of $M$ is separated into: 1) the motion relative to the orbital plane and 2) the inertial motion of the orbital plane. We start the dynamical analysis describing the time evolution of this last motion. 


\section{Evolution of the orbital plane}

The attitude of the frame $O x_{p} y_{p} z_{p}$ in the inertial space is described by the quaternion $\mathbf{n}$ given by (3) or the matrix $P_{0}$ given by (4). Both are constant in the Keplerian motion and change with time in the perturbed motion.

The time evolution of the quaternion $\mathbf{n}$ is given by:

$$
\frac{\mathrm{d} \mathbf{n}}{\mathrm{d} \tau}=\frac{1}{2} \mathbf{n}\left(2 \hat{\omega}_{01}\right)
$$

where $2 \hat{\omega}_{01}$ is the vectorial quaternion associated with the angular velocity $\boldsymbol{\omega}_{01}$ of the orbital plane. The components of $\boldsymbol{\omega}_{01}$ in the frame $O x_{p} y_{p} z_{p}$ are:

$$
\boldsymbol{\omega}_{01}=\frac{r}{h}\left(a_{p z}\right)\left(\cos \sigma \boldsymbol{u}_{1}+\sin \sigma \boldsymbol{u}_{2}\right)
$$

Taking into account these components, equations (16) take the form:

$$
\begin{aligned}
\frac{\mathrm{d} \eta_{1}}{\mathrm{~d} \tau} & =\frac{1}{2} \frac{r}{h}\left(a_{p z}\right)\left\{\eta_{4} \cos \sigma-\eta_{3} \sin \sigma\right\} \\
\frac{\mathrm{d} \eta_{2}}{\mathrm{~d} \tau} & =\frac{1}{2} \frac{r}{h}\left(a_{p z}\right)\left\{\eta_{3} \cos \sigma+\eta_{4} \sin \sigma\right\} \\
\frac{\mathrm{d} \eta_{3}}{\mathrm{~d} \tau} & =\frac{1}{2} \frac{r}{h}\left(a_{p z}\right)\left\{-\eta_{2} \cos \sigma+\eta_{1} \sin \sigma\right\} \\
\frac{\mathrm{d} \eta_{4}}{\mathrm{~d} \tau} & =\frac{1}{2} \frac{r}{h}\left(a_{p z}\right)\left\{-\eta_{1} \cos \sigma-\eta_{2} \sin \sigma\right\}
\end{aligned}
$$

To calculate the perturbation component $a_{p z}$ the position of the orbital frame $O x y z$ is necessary. Let $\mathbf{q}$ the quaternion:

$$
\mathbf{q}=\varepsilon_{4}+\left(\varepsilon_{1}, \varepsilon_{2}, \varepsilon_{3}\right)
$$

that determines the attitude of frame $O x y z$ in the inertial space. The frame $O x y z$ can be obtained, from the frame $O x_{p} y_{p} z_{p}$, through a rotation of angle $\sigma$ around $O z$ :

$$
\lfloor\boldsymbol{i}, \boldsymbol{j}, \boldsymbol{k}\rceil=\left\lfloor\boldsymbol{u}_{1}, \boldsymbol{u}_{2}, \boldsymbol{k}\right\rceil R_{3}(\sigma) \quad \text { where } \quad R_{3}(\sigma)=\left(\begin{array}{ccc}
\cos \sigma & -\sin \sigma & 0 \\
\sin \sigma & \cos \sigma & 0 \\
0 & 0 & 1
\end{array}\right)
$$

Let $\hat{\sigma}$ the unitary quaternion associated with the matrix $R_{3}(\sigma)$ :

$$
\hat{\sigma}=\cos \left(\frac{\sigma}{2}\right)+\left(0,0, \sin \left(\frac{\sigma}{2}\right)\right)
$$

The quaternion $\mathbf{q}$ can be obtained as the product $\mathbf{q}=\mathbf{n} \hat{\sigma}$ that expressed in components takes the form

$$
\begin{array}{ll}
\varepsilon_{1}=\eta_{1} \cos \frac{\sigma}{2}+\eta_{2} \sin \frac{\sigma}{2}, & \varepsilon_{2}=\eta_{2} \cos \frac{\sigma}{2}-\eta_{1} \sin \frac{\sigma}{2} \\
\varepsilon_{3}=\eta_{3} \cos \frac{\sigma}{2}+\eta_{4} \sin \frac{\sigma}{2}, & \varepsilon_{4}=\eta_{4} \cos \frac{\sigma}{2}-\eta_{3} \sin \frac{\sigma}{2}
\end{array}
$$

\section{Motion relative to the orbital plane}

If we focus the analysis on the motion relative to the orbital plane, the dynamic state of the particle $M$ is given by $(\tau, r, \sigma, \dot{r}, \dot{\sigma})$ since $(r, \sigma)$ are polar coordinates of $M$ in the ideal frame $O x_{p} y_{p} z_{p}$. In DROMO $\sigma$ will be taken as the independent variable and $\tau$ will be considered as a dependent variable. We calculate the time derivative of different magnitudes in the ideal frame. 
In order to do that we use a particular version of the Coriolis theorem:

$$
\left.\frac{\mathrm{d} \boldsymbol{A}}{\mathrm{d} \tau}\right|_{(1)}=\left.\frac{\mathrm{d} \boldsymbol{A}}{\mathrm{d} \tau}\right|_{(0)}+\boldsymbol{\omega}_{01} \times\left.\boldsymbol{A} \Leftrightarrow \frac{\mathrm{d} \boldsymbol{A}}{\mathrm{d} \sigma}\right|_{(1)}=\left.\frac{\mathrm{d} \boldsymbol{A}}{\mathrm{d} \sigma}\right|_{(0)}+\boldsymbol{\Omega}_{01} \times \boldsymbol{A}
$$

where $\Omega_{01}$ is the vector:

$$
\boldsymbol{\Omega}_{01}=\boldsymbol{\omega}_{01} \frac{r^{2}}{h}=\frac{r^{3}}{h^{2}} a_{p z} \boldsymbol{i}
$$

The $\sigma$-derivative performed in the ideal frame assume that the unit vectors $\left(\boldsymbol{u}_{1}, \boldsymbol{u}_{2}, \boldsymbol{k}\right)$ do not change when time goes on. In what follows we calculate the $\sigma$-derivative of several magnitudes of interest in the motion relative to the orbital plane.

\section{Velocity vector $v$. Angular momentum $h$. Eccentricity vector $e$}

Velocity vector $\boldsymbol{v}$ Obviously:

$\left.\frac{\mathrm{d} \boldsymbol{v}}{\mathrm{d} \tau}\right|_{(1)}=-\frac{\boldsymbol{i}}{r^{2}}+\left.\boldsymbol{a}_{p} \Rightarrow \frac{\mathrm{d} \boldsymbol{v}}{\mathrm{d} \sigma}\right|_{(1)}=\frac{1}{h}\left(-\boldsymbol{i}+r^{2} \boldsymbol{a}_{p}\right), \quad$ and $\quad \boldsymbol{\Omega}_{01} \times \boldsymbol{v}=\Omega_{01} \boldsymbol{i} \times \boldsymbol{v}=\frac{\Omega_{01}}{r} \boldsymbol{h}=\frac{r^{2}}{h} a_{p z} \boldsymbol{k}$

From the Coriolis theorem (22) we deduce:

$$
\left.\frac{\mathrm{d} \boldsymbol{v}}{\mathrm{d} \sigma}\right|_{(0)}=\frac{1}{h}\left(-\boldsymbol{i}+r^{2} \boldsymbol{a}_{p \|}\right)
$$

where $\boldsymbol{a}_{p \|}$ is the component of $\boldsymbol{a}_{p}$ parallel to the orbital plane:

$$
\boldsymbol{a}_{p \|}=\boldsymbol{a}_{p}-a_{p z} \boldsymbol{k}
$$

Angular momentum $\boldsymbol{h}$ The angular momentum $\boldsymbol{h}$ verifies:

$$
\left.\frac{\mathrm{d} \boldsymbol{h}}{\mathrm{d} \sigma}\right|_{(1)}=\frac{r^{2}}{h} \boldsymbol{x} \times \boldsymbol{a}_{p}=\frac{r^{3}}{h} \boldsymbol{i} \times \boldsymbol{a}_{p} \quad \text { and, on the other hand } \quad \boldsymbol{\Omega}_{01} \times \boldsymbol{h}=\Omega_{01} h \boldsymbol{i} \times \boldsymbol{k}=-\frac{r^{3}}{h} a_{p z} \boldsymbol{j}
$$

From the Coriolis theorem (22) we deduce:

$$
\left.\frac{\mathrm{d} \boldsymbol{h}}{\mathrm{d} \sigma}\right|_{(0)}=\frac{r^{3}}{h} a_{p y} \boldsymbol{k}=\frac{r^{3}}{h} \boldsymbol{i} \times \boldsymbol{a}_{p \|}, \quad \Rightarrow \quad \frac{\mathrm{d} h}{\mathrm{~d} \sigma}=\frac{r^{3}}{h} a_{p y}
$$

Thus, the equations that govern the motion relative to the orbital plane are identical to the equations that govern the inertial motion, if we change the perturbation acceleration $\boldsymbol{a}_{p}$ by its component parallel to the orbital plane $\boldsymbol{a}_{p \|}$.

Eccentricity vector $e$ In non-dimensional variables the eccentricity vector and its $\sigma$-derivative are given by:

$$
\boldsymbol{e}=-\boldsymbol{i}-\boldsymbol{h} \times \boldsymbol{v},\left.\quad \frac{\mathrm{d} \boldsymbol{e}}{\mathrm{d} \sigma}\right|_{(0)}=-\left.\frac{\mathrm{d} \boldsymbol{i}}{\mathrm{d} \sigma}\right|_{(0)}-\left.\frac{\mathrm{d} \boldsymbol{h}}{\mathrm{d} \sigma}\right|_{(0)} \times \boldsymbol{v}-\boldsymbol{h} \times\left.\frac{\mathrm{d} \boldsymbol{v}}{\mathrm{d} \sigma}\right|_{(0)}
$$

The hodograph equation:

$$
\boldsymbol{v}=\frac{1}{h}(\boldsymbol{j}+\boldsymbol{k} \times \boldsymbol{e})
$$

permits to calculate the right hand side of this equation. Taking into account the following $\sigma$-derivatives:

$$
\left.\frac{\mathrm{d} \boldsymbol{i}}{\mathrm{d} \sigma}\right|_{(0)}=\boldsymbol{j},\left.\quad \frac{\mathrm{d} \boldsymbol{h}}{\mathrm{d} \sigma}\right|_{(0)} \times \boldsymbol{v}=-\frac{r^{3}}{h^{2}} a_{p y}(\boldsymbol{i}+\boldsymbol{e}), \quad \boldsymbol{h} \times\left.\frac{\mathrm{d} \boldsymbol{v}}{\mathrm{d} \sigma}\right|_{(0)}=-\boldsymbol{j}+r^{2} \boldsymbol{k} \times \boldsymbol{a}_{p \|}
$$


we obtain

$$
\left.\frac{\mathrm{d} \boldsymbol{e}}{\mathrm{d} \sigma}\right|_{(0)}=\frac{r^{3}}{h^{2}}\left(a_{p y}[\boldsymbol{i}+\boldsymbol{e}]-\frac{h^{2}}{r} \boldsymbol{k} \times \boldsymbol{a}_{p \|}\right)
$$

In order to lighten notation we introduce the vectors $\boldsymbol{b}_{p \|}$ and $\boldsymbol{a}_{f}$, defined by:

$$
\boldsymbol{b}_{p \|}=\frac{r^{3}}{h^{2}} \boldsymbol{a}_{p \|}, \quad \boldsymbol{a}_{f}=\frac{h^{2}}{r} \boldsymbol{b}_{p \|}+b_{p y} \boldsymbol{j}
$$

The $\sigma$-derivative of the eccentricity vector $\boldsymbol{e}$ takes the form:

$$
\left.\frac{\mathrm{d} \boldsymbol{e}}{\mathrm{d} \sigma}\right|_{(0)}=\boldsymbol{a}_{f} \times \boldsymbol{k}+b_{p y} \boldsymbol{e}
$$

\section{EQUATIONS OF MOTION}

To describe the motion relative to the orbital plane we take the following variables to define the dynamic state of the particle: $\left(\sigma ; \tau, \zeta_{1}, \zeta_{2}, \zeta_{3}\right)$. Here, the variable $\zeta_{3}=1 / h$ is the inverse of the angular momentum $h$ and $\left(\zeta_{1}, \zeta_{2}\right)$ are the coordinates of the eccentricity vector in the ideal frame: $\boldsymbol{e}=\zeta_{1} \boldsymbol{u}_{1}+\zeta_{2} \boldsymbol{u}_{2}$. Therefore, the $\sigma$-derivative of the eccentricity vector will be:

$$
\left.\frac{\mathrm{d} \boldsymbol{e}}{\mathrm{d} \sigma}\right|_{(0)}=\frac{\mathrm{d} \zeta_{1}}{\mathrm{~d} \sigma} \boldsymbol{u}_{1}+\frac{\mathrm{d} \zeta_{2}}{\mathrm{~d} \sigma} \boldsymbol{u}_{2}
$$

The vectorial equation (27) will be projected on the basis $\left(\boldsymbol{u}_{1}, \boldsymbol{u}_{2}\right)$ and by taking into account the relations:

$$
\boldsymbol{i}=+\cos \sigma \boldsymbol{u}_{1}+\sin \sigma \boldsymbol{u}_{2}, \quad \boldsymbol{j}=-\sin \sigma \boldsymbol{u}_{1}+\cos \sigma \boldsymbol{u}_{2}
$$

the following equations, which determine the evolution of the coordinates $\left(\zeta_{1}, \zeta_{2}\right)$, can be obtained:

$$
\begin{aligned}
& \frac{\mathrm{d} \zeta_{1}}{\mathrm{~d} \sigma}=+\boldsymbol{a}_{f} \cdot \boldsymbol{u}_{2}+b_{p y} \zeta_{1} \\
& \frac{\mathrm{d} \zeta_{2}}{\mathrm{~d} \sigma}=-\boldsymbol{a}_{f} \cdot \boldsymbol{u}_{1}+b_{p y} \zeta_{2}
\end{aligned}
$$

Note that $\left(\zeta_{1}, \zeta_{2}\right)$ are first integrals of the Keplerian motion, because they keep constant values when the perturbation $\boldsymbol{a}_{p}$ vanishes.

From equation (25) we get:

$$
\frac{\mathrm{d} \zeta_{3}}{\mathrm{~d} \sigma}=-r^{3} \zeta_{3}^{3} a_{p y}
$$

and finally, from the previous kinematic relations we obtain:

$$
\frac{\mathrm{d} \tau}{\mathrm{d} \sigma}=r^{2} \zeta_{3}
$$

In summary, in terms of the DROMO variables $\left(\sigma ; \tau, \zeta_{1}, \zeta_{2}, \zeta_{3}\right)$ the motion of the particle $M$ relative to the orbital plane is governed by equations (29-41). The right hand sides of these equations should be expressed in terms of $\left(\sigma ; \tau, \zeta_{1}, \zeta_{2}, \zeta_{3}\right)$, as we show in the following section.

\section{Additional simplifications}

Note that $(r, \sigma)$ are polar coordinates of $M$ in the departure perifocal frame $O x_{p} y_{p} z_{p}$; as a consequence the relative velocity $\boldsymbol{v}$ turns out to be:

$$
\boldsymbol{v}=\frac{\mathrm{d} r}{\mathrm{~d} \tau} \boldsymbol{i}+\frac{h}{r} \boldsymbol{j}
$$


and the eccentricity vector, $\boldsymbol{e}=-\boldsymbol{i}-\boldsymbol{h} \times \boldsymbol{v}$, can be expressed in two different forms:

$$
\boldsymbol{e}=\left(\frac{h^{2}}{r}-1\right) \boldsymbol{i}-h \frac{\mathrm{d} r}{\mathrm{~d} \tau} \boldsymbol{j} \quad \text { and also } \quad \boldsymbol{e}=\zeta_{1} \boldsymbol{u}_{1}+\zeta_{2} \boldsymbol{u}_{2}
$$

Taking into account equations (28) we obtain the following relations

$$
\begin{aligned}
& \zeta_{1}=\left(\frac{h^{2}}{r}-1\right) \cos \sigma+h \frac{\mathrm{d} r}{\mathrm{~d} \tau} \sin \sigma \\
& \zeta_{2}=\left(\frac{h^{2}}{r}-1\right) \sin \sigma-h \frac{\mathrm{d} r}{\mathrm{~d} \tau} \cos \sigma
\end{aligned}
$$

The inverse relations of these equations are

$$
\begin{aligned}
\frac{1}{r} & =\zeta_{3}^{2} s \quad \text { where } \quad s=1+\zeta_{1} \cos \sigma+\zeta_{2} \sin \sigma \\
\frac{\mathrm{d} r}{\mathrm{~d} \tau} & =\zeta_{3}\left(\zeta_{1} \sin \sigma-\zeta_{2} \cos \sigma\right)
\end{aligned}
$$

and they provide the range $r$ and the range rate $\dot{r}$ in terms of DROMO variable $\left(\sigma ; \tau, \zeta_{1}, \zeta_{2}, \zeta_{3}\right)$. In order to simplify the notation we introduced the amount $s=1+\zeta_{1} \cos \sigma+\zeta_{2} \sin \sigma$.

\section{Summary of equations}

Joining the equation that govern the motion relative to the orbital plane and the inertial motion of this plane we obtain a closed set of equations that describe the inertial motion of the particle $M$. In summary, the equations that govern the motion are:

$$
\begin{aligned}
\frac{\mathrm{d} \tau}{\mathrm{d} \sigma} & =\frac{1}{\zeta_{3}^{3} s^{2}} \\
\frac{\mathrm{d} \zeta_{1}}{\mathrm{~d} \sigma} & =+s \sin \sigma\left(\tilde{a}_{p x}\right)+\left(\zeta_{1}+(1+s) \cos \sigma\right)\left(\tilde{a}_{p y}\right) \\
\frac{\mathrm{d} \zeta_{2}}{\mathrm{~d} \sigma} & =-s \cos \sigma\left(\tilde{a}_{p x}\right)+\left(\zeta_{2}+(1+s) \sin \sigma\right)\left(\tilde{a}_{p y}\right) \\
\frac{\mathrm{d} \zeta_{3}}{\mathrm{~d} \sigma} & =-\zeta_{3}\left(\tilde{a}_{p y}\right) \\
\frac{\mathrm{d} \eta_{1}}{\mathrm{~d} \sigma} & =\frac{1}{2} \tilde{a}_{p z}\left\{\eta_{4} \cos \sigma-\eta_{3} \sin \sigma\right\} \\
\frac{\mathrm{d} \eta_{2}}{\mathrm{~d} \sigma} & =\frac{1}{2} \tilde{a}_{p z}\left\{\eta_{3} \cos \sigma+\eta_{4} \sin \sigma\right\} \\
\frac{\mathrm{d} \eta_{3}}{\mathrm{~d} \sigma} & =\frac{1}{2} \tilde{a}_{p z}\left\{-\eta_{2} \cos \sigma+\eta_{1} \sin \sigma\right\} \\
\frac{\mathrm{d} \eta_{4}}{\mathrm{~d} \sigma} & =\frac{1}{2} \tilde{a}_{p z}\left\{-\eta_{1} \cos \sigma-\eta_{2} \sin \sigma\right\}
\end{aligned}
$$

$$
\begin{aligned}
s & =1+\zeta_{1} \cos \sigma+\zeta_{2} \sin \sigma \\
\frac{1}{r} & =\zeta_{3}^{2} s \\
\frac{\mathrm{d} r}{\mathrm{~d} \tau} & =\zeta_{3}\left(\zeta_{1} \sin \sigma-\zeta_{2} \cos \sigma\right) \\
\varepsilon_{1} & =\eta_{1} \cos \frac{\sigma}{2}+\eta_{2} \sin \frac{\sigma}{2} \\
\varepsilon_{2} & =\eta_{2} \cos \frac{\sigma}{2}-\eta_{1} \sin \frac{\sigma}{2} \\
\varepsilon_{3} & =\eta_{3} \cos \frac{\sigma}{2}+\eta_{4} \sin \frac{\sigma}{2} \\
\varepsilon_{4} & =\eta_{4} \cos \frac{\sigma}{2}-\eta_{3} \sin \frac{\sigma}{2} \\
\tilde{a}_{p x} & =\frac{a_{p x}}{\zeta_{3}^{4} s^{3}}, \tilde{a}_{p y}=\frac{a_{p y}}{\zeta_{3}^{4} s^{3}}, \tilde{a}_{p z}=\frac{a_{p z}}{\zeta_{3}^{4} s^{3}} \\
1 & =\eta_{1}^{2}+\eta_{2}^{2}+\eta_{3}^{2}+\eta_{4}^{2}
\end{aligned}
$$

and they must be integrated with the additional relations shown on the right side of the page, starting from the appropriate initial conditions.

\section{Initial conditions}

Let us assume that we know the dynamical state of the system in the initial time $t_{0}$; to fix ideas we assume that we know the initial conditions $\left(\boldsymbol{x}_{0}, \boldsymbol{v}_{0}\right)$, that is, the state vector in the inertial frame (in non-dimensional form).

From these data it is fairly straightforward to calculate the vectors:

$$
\boldsymbol{h}_{0}=\boldsymbol{x}_{0} \times \boldsymbol{v}_{0}, \quad \boldsymbol{e}_{0}=-\frac{\boldsymbol{x}_{0}}{\left|\boldsymbol{x}_{0}\right|}-\boldsymbol{h}_{0} \times \boldsymbol{v}_{0}
$$


which permit to determine the unit vectors of the departure perifocal frame $O x_{p} y_{p} z_{p}$ :

$$
\boldsymbol{k}(0)=\frac{\boldsymbol{h}_{0}}{\left|\boldsymbol{h}_{0}\right|}, \quad \boldsymbol{u}_{1}(0)=\frac{\boldsymbol{e}_{0}}{\left|\boldsymbol{e}_{0}\right|}, \quad \boldsymbol{u}_{2}(0)=\boldsymbol{k}(0) \times \boldsymbol{u}_{1}(0)
$$

and also the unit vector of the orbital frame $O x y z$ :

$$
\boldsymbol{i}(0)=\frac{\boldsymbol{x}_{0}}{\left|\boldsymbol{x}_{0}\right|}, \quad \boldsymbol{j}(0)=\boldsymbol{k}(0) \times \boldsymbol{i}(0)
$$

The unit vectors $\left(\boldsymbol{u}_{1}(0), \boldsymbol{u}_{2}(0), \boldsymbol{k}(0)\right)$ determine the initial value of the matrix $P_{0}$ described in (4) and from this matrix is also straightforward to calculate the initial values of the Euler parameters $\left(\eta_{1_{0}}, \eta_{2_{0}}, \eta_{3_{0}}, \eta_{4_{0}}\right)$.

The initial value of the $\sigma$ angle (true anomaly) is given by:

$$
\cos \sigma_{0}=\boldsymbol{i}(0) \cdot \boldsymbol{u}_{1}(0), \quad \sin \sigma_{0}=-\boldsymbol{j}(0) \cdot \boldsymbol{u}_{1}(0)
$$

Finally, he initial values of the DROMO variables are:

$$
\begin{aligned}
& \sigma(0)=\sigma_{0}, \quad \tau(0)=0, \quad \zeta_{1}(0)=e_{0}, \quad \zeta_{2}(0)=0, \quad \zeta_{3}(0)=1 / h_{0} \\
& \eta_{1}(0)=\eta_{1_{0}}, \quad \eta_{2}(0)=\eta_{2_{0}}, \quad \eta_{3}(0)=\eta_{3_{0}}, \quad \eta_{4}(0)=\eta_{4_{0}}
\end{aligned}
$$

\section{Singularities}

The procedure described to obtain the initial values of the DROMO variables fails when $h_{0}=0$ or $e_{0}=0$. In the first case the singularity cannot be prevented. In order to avoid it a reformulation of variables is mandatory. We will not describe here such a reformulation of variables which will be presented in further works.

In the second case, $e_{0}=0$, the singularity can be avoided. In effect, if we expand the eccentricity vector around $t_{0}$ we have:

$$
\boldsymbol{e}(t)=\boldsymbol{e}_{0}+\left.\frac{\mathrm{d} \boldsymbol{e}}{\mathrm{d} t}\right|_{t_{0}}\left(t-t_{0}\right)+\ldots
$$

To avoid discontinuities in the ephemeris provided by the numerical integration, the unit vector $\boldsymbol{u}_{1}(0)$ should be selected in the direction pointed out by the derivative of $e$ which is given by:

$$
\left.\frac{\mathrm{d} \boldsymbol{e}}{\mathrm{d} t}\right|_{t_{0}}=\left.h_{0}\left\{2 a_{p y} \boldsymbol{i}-a_{p x} \boldsymbol{j}\right\}\right|_{t_{0}}
$$

It depends on the perturbation on the initial time.

\section{FROM DROMO VARIABLES TO "STATE VECTOR"}

Let us assume that the dynamical state of the system is known in terms of the DROMO variables:

$$
\left(\sigma ; \tau, \zeta_{1}, \zeta_{2}, \zeta_{3}, \eta_{1}, \eta_{2}, \eta_{3}, \eta_{4}\right)
$$

The aim is to determine the classical state vector $\left(\boldsymbol{x}_{1}, \boldsymbol{v}_{1}\right)$-here dimensional values-of the particle through their coordinates in the inertial frame $O x_{1} y_{1} z_{1}$. Obviously, the physical time $t$ is determined from the nondimensional time $\tau$ :

$$
t-t_{0}=\tau / \omega_{c}
$$

Vector $\boldsymbol{x}_{1}$ can be expressed in the departure perifocal frame $O x_{p} y_{p} z_{p}$ as:

$$
\boldsymbol{x}_{1}=L_{c} r\left(\cos \sigma \boldsymbol{u}_{1}+\sin \sigma \boldsymbol{u}_{2}\right)=\frac{L_{c}}{\zeta_{3}^{2} s}\left(\cos \sigma \boldsymbol{u}_{1}+\sin \sigma \boldsymbol{u}_{2}\right)
$$


In terms of its inertial coordinates it takes the form:

$$
\boldsymbol{x}_{1}=\frac{L_{c}}{\zeta_{3}^{2} s}\left\lfloor\boldsymbol{i}_{1}, \boldsymbol{j}_{1}, \boldsymbol{k}_{1}\right\rceil P_{0}\left(\begin{array}{c}
\cos \sigma \\
\sin \sigma \\
0
\end{array}\right)
$$

where the matrix $P_{0}$ is described in (4).

From the hodograph equation, vector $\boldsymbol{v}_{1}$ can be expressed in the departure perifocal frame $O x_{p} y_{p} z_{p}$ as:

$$
\boldsymbol{v}_{1}=\left(\omega_{c} L_{c}\right) \zeta_{3}\left\{\boldsymbol{j}+\zeta_{1} \boldsymbol{u}_{2}-\zeta_{2} \boldsymbol{u}_{1}\right\}=\left(\omega_{c} L_{c}\right) \zeta_{3}\left\{-\left(\sin \sigma+\zeta_{2}\right) \boldsymbol{u}_{1}+\left(\cos \sigma+\zeta_{1}\right) \boldsymbol{u}_{2}\right\}
$$

We introduce the amounts $\left(V_{1}, V_{2}\right)$ defined as:

$$
V_{1}=-\zeta_{3}\left(\sin \sigma+\zeta_{2}\right), \quad V_{2}=\zeta_{3}\left(\cos \sigma+\zeta_{1}\right)
$$

In terms of its inertial coordinates vector $\boldsymbol{v}_{1}$ takes the form:

$$
\boldsymbol{v}_{1}=\left(\omega_{c} L_{c}\right)\left\lfloor\boldsymbol{i}_{1}, \boldsymbol{j}_{1}, \boldsymbol{k}_{1}\right\rceil P_{0}\left(\begin{array}{c}
V_{1} \\
V_{2} \\
0
\end{array}\right)
$$

\section{FROM DROMO VARIABLES TO CLASSICAL ELEMENTS}

In this section we show how to pass from the DROMO variables to the classical elementos of the osculating orbit. Obviously the eccentricity is:

$$
e=\sqrt{\zeta_{1}^{2}+\zeta_{2}^{2}}
$$

The kinetic, the potential and the total Keplerian energies are given by:

$$
T=\frac{1}{2} m \omega_{c}^{2} L_{c}^{2} \zeta_{3}^{2}\left(e^{2}-1+2 s\right), \quad V=-m \omega_{c}^{2} L_{c}^{2} \zeta_{3}^{2} s, \quad E=\frac{1}{2} m \omega_{c}^{2} L_{c}^{2} \zeta_{3}^{2}\left(\zeta_{1}^{2}+\zeta_{2}^{2}-1\right)
$$

and the semi-major axis turns out to be:

$$
a=-\frac{L_{c}}{\zeta_{3}^{2}\left(\zeta_{1}^{2}+\zeta_{2}^{2}-1\right)}
$$

Let $\beta$ be the angle that the eccentricity vector forms with the $O x_{p}$ axis; its value can be calculated from:

$$
\beta=\arctan \left(\frac{\zeta_{2}}{\zeta_{1}}\right)
$$

Notice that the true anomaly of $M$ in the osculating orbit is $\theta=\sigma-\beta$.

From the values of the Euler parameters $\left(\eta_{1}, \eta_{2}, \eta_{3}, \eta_{4}\right)$ the following expressions:

$$
i=2 \arccos \left(\sqrt{\eta_{3}^{2}+\eta_{4}^{2}}\right), \quad \Omega+\tilde{\omega}=2 \arctan \left(\frac{\eta_{3}}{\eta_{4}}\right), \quad \Omega-\tilde{\omega}=2 \arctan \left(\frac{\eta_{2}}{\eta_{1}}\right)
$$

provide the values of inclination, $i$, the longitud of the ascending node, $\Omega$, and the argument of the $O x_{p}$ axis, $\tilde{\omega}$; note that the argument of pericenter $\omega$ is given by:

$$
\omega=\tilde{\omega}+\beta
$$

Finally, the time of pericenter passage depends on the kind of the osculating orbit. If we assume that the orbit is elliptical $e<1$ the procedure is as follows: 
1. we calculate the true and the eccentric anomalies from relations:

$$
\theta=\sigma-\beta, \quad \sin \mathcal{E}=\frac{\sqrt{1-e^{2}} \sin \theta}{1+e \cos \theta}, \quad \cos \mathcal{E}=\frac{\cos \theta+e}{1+e \cos \theta}
$$

2. we calculate the time of pericenter passage from relation:

$$
t_{p}=t-\frac{\mathcal{E}-e \sin \mathcal{E}}{\zeta_{3}^{3}\left(\zeta_{1}^{2}+\zeta_{2}^{2}-1\right)^{3 / 2}} \frac{1}{\omega_{c}}
$$

Similar procedures can be used for parabolic or hyperbolic osculating orbits. Even it is posible to use an universal formulation including the three kind of orbits.

\section{COMPARISON WITH DEPRIT'S EQUATIONS}

In 1975 A. Deprit ${ }^{3}$ deduced a set of equations based on the ideal frame concept. In this section we describe the analysis of Deprit considering the DROMO formulation. We start the analysis rewriting the hodograph equation in the form:

$$
\boldsymbol{v}=\frac{1}{h} \boldsymbol{j}+\boldsymbol{z}, \quad \text { where } \quad \boldsymbol{z}=\frac{1}{h}(\boldsymbol{k} \times \boldsymbol{e})
$$

In the motion relative to the orbital plane:

$$
\left.\frac{\mathrm{d} \boldsymbol{z}}{\mathrm{d} \sigma}\right|_{(0)}=\left.\frac{\mathrm{d} \boldsymbol{v}}{\mathrm{d} \sigma}\right|_{(0)}-\left.\frac{1}{h} \frac{\mathrm{d} \boldsymbol{j}}{\mathrm{d} \sigma}\right|_{(0)}+\left.\frac{1}{h^{2}} \frac{\mathrm{d} h}{\mathrm{~d} \sigma}\right|_{(0)} \boldsymbol{j}
$$

and taking into account the derivatives previously calculated we obtain:

$$
\left.\frac{\mathrm{d} \boldsymbol{z}}{\mathrm{d} \sigma}\right|_{(0)}=\frac{1}{h} \boldsymbol{a}_{f}
$$

However, the vector $\boldsymbol{z}$ can be expressed in the actual perifocal frame $\left\lfloor\boldsymbol{e}_{1}, \boldsymbol{e}_{2}, \boldsymbol{k}\right\rceil$ (do not confuse withe the departure perifocal frame) as follows:

$$
\boldsymbol{z}=\left.\frac{e}{h} \boldsymbol{e}_{2} \Rightarrow \frac{\mathrm{d} \boldsymbol{z}}{\mathrm{d} \sigma}\right|_{(0)}=\frac{\mathrm{d}}{\mathrm{d} \sigma}\left(\frac{e}{h}\right) \boldsymbol{e}_{2}+\left.\frac{e}{h} \frac{\mathrm{d} \boldsymbol{e}_{2}}{\mathrm{~d} \sigma}\right|_{(0)}
$$

This relation is introduced in (37) and then a dot product with $\boldsymbol{e}_{2}$ is carried out. We obtain:

$$
\frac{\mathrm{d}}{\mathrm{d} \sigma}\left(\frac{e}{h}\right)=\frac{1}{h} \boldsymbol{a}_{f} \cdot \boldsymbol{e}_{2}
$$

and this equation is the equivalent, in DROMO's formulation, to the equation (70) in Deprit's paper. Taking into account this result, equation (37) provides:

$$
\left.\frac{\mathrm{d} \boldsymbol{e}_{2}}{\mathrm{~d} \sigma}\right|_{(0)}=\frac{1}{e}\left(\boldsymbol{a}_{f}-\left[\boldsymbol{a}_{f} \cdot \boldsymbol{e}_{2}\right] \boldsymbol{e}_{2}\right)=+\frac{1}{e}\left(\boldsymbol{a}_{f} \cdot \boldsymbol{e}_{1}\right) \boldsymbol{e}_{1}
$$

As a consequence:

$$
\left.\frac{\mathrm{d} \boldsymbol{e}_{2}}{\mathrm{~d} \sigma}\right|_{(0)}=\frac{\mathrm{d} \beta}{\mathrm{d} \sigma} \boldsymbol{k} \times \boldsymbol{e}_{2}, \quad \Rightarrow \quad \frac{\mathrm{d} \beta}{\mathrm{d} \sigma}=-\frac{1}{e}\left(\boldsymbol{a}_{f} \cdot \boldsymbol{e}_{1}\right)
$$

By expanding the left hand side of (38) we obtain:

$$
\frac{\mathrm{d} e}{\mathrm{~d} \sigma}=\boldsymbol{a}_{f} \cdot \boldsymbol{e}_{2}+e b_{p y}
$$


but

$$
\left.\frac{\mathrm{d} \boldsymbol{e}}{\mathrm{d} \sigma}\right|_{(0)}=\left.\frac{\mathrm{d}\left(e \boldsymbol{e}_{1}\right)}{\mathrm{d} \sigma}\right|_{(0)}=\frac{\mathrm{d} e}{\mathrm{~d} \sigma} \boldsymbol{e}_{1}+\left.e \frac{\mathrm{d} \boldsymbol{e}_{1}}{\mathrm{~d} \sigma}\right|_{(0)}
$$

and since the $\sigma$-derivative on the right hand side of this relation are known we obtain:

$$
\left.\frac{\mathrm{d} \boldsymbol{e}}{\mathrm{d} \sigma}\right|_{(0)}=\left(\boldsymbol{a}_{f} \cdot \boldsymbol{e}_{2}\right) \boldsymbol{e}_{1}-\left(\boldsymbol{a}_{f} \cdot \boldsymbol{e}_{1}\right) \boldsymbol{e}_{2}+b_{p y} \boldsymbol{e}
$$

Finally the identity

$$
\left(a_{f} \cdot e_{2}\right) e_{1}-\left(a_{f} \cdot e_{1}\right) e_{2}=a_{f} \times\left(e_{1} \times e_{2}\right)
$$

leads to equation (27) which governs the evolution of the eccentricity vector.

Let $\boldsymbol{T}$ be the vector:

$$
\boldsymbol{T}=\frac{1}{h} \boldsymbol{e} \quad \text { whose } \sigma \text {-derivative is }\left.\quad \frac{\mathrm{d} \boldsymbol{T}}{\mathrm{d} \sigma}\right|_{(0)}=\left.\frac{1}{h} \frac{\mathrm{d} \boldsymbol{e}}{\mathrm{d} \sigma}\right|_{(0)}-\boldsymbol{e} \frac{1}{h^{2}} \frac{\mathrm{d} h}{\mathrm{~d} \sigma}
$$

Since the $\sigma$-derivatives in the right hand side are know we get

$$
\left.\frac{\mathrm{d} \boldsymbol{T}}{\mathrm{d} \sigma}\right|_{(0)}=\frac{1}{h}\left(\boldsymbol{a}_{f} \times \boldsymbol{k}+b_{p y} \boldsymbol{e}\right)-\left.\frac{1}{h} \boldsymbol{e} b_{p y} \quad \Rightarrow \quad \frac{\mathrm{d} \boldsymbol{T}}{\mathrm{d} \sigma}\right|_{(0)}=\frac{1}{h}\left(\boldsymbol{a}_{f} \times \boldsymbol{k}\right)
$$

Let $\left(q_{1}, q_{2}\right)$ be the coordinates of $\boldsymbol{T}$ in the departure perifocal frame $\left(\boldsymbol{u}_{1}, \boldsymbol{u}_{2}\right)$ :

$$
\boldsymbol{T}=q_{1} \boldsymbol{u}_{1}+q_{2} \boldsymbol{u}_{2}
$$

The evolution of $\left(q_{1}, q_{2}\right)$ are given by

$$
\begin{aligned}
& \frac{\mathrm{d} q_{1}}{\mathrm{~d} \sigma}=+\frac{1}{h} \boldsymbol{a}_{f} \cdot \boldsymbol{u}_{2} \\
& \frac{\mathrm{d} q_{2}}{\mathrm{~d} \sigma}=-\frac{1}{h} \boldsymbol{a}_{f} \cdot \boldsymbol{u}_{1}
\end{aligned}
$$

and they are equivalent, inside the DROMO formulation, to the equation (76-77) in Deprit's paper. Note that, the original formulation of DROMO in paper ${ }^{2}$ involves these variables $\left(q_{1}, q_{2}\right)$.

Despite similarities between Deprit and DROMO formulations we should underline the main differences between them:

1. Equations (42-43) are not exactly equations (76-77) in Deprit's paper; they are linear combinations of them. In effect, reference 3 use the componen $(\mathcal{C}, \mathcal{S})$ of $\boldsymbol{T}$ in the departure frame $O x_{0} y_{0} z_{0}$ which are related with the $\left(q_{1}, q_{2}\right)$ coordinates by equations:

$$
\left(\begin{array}{l}
\mathcal{C} \\
\mathcal{S}
\end{array}\right)=\left(\begin{array}{cc}
\cos \sigma & \sin \sigma \\
-\sin \sigma & \cos \sigma
\end{array}\right)\left(\begin{array}{l}
q_{1} \\
q_{2}
\end{array}\right)
$$

2. reference 3 use time $t$ as independent variable and it is focused on elliptical orbits. DROMO, however, use the angle $\sigma$ as independent variable and this permit an universal formulation which can be used in elliptic, parabolic or hyperbolic orbits. This is an important advantage in some optimization problems which appears in interplanetary trajectories (see reference 20). 


\section{NUMERICAL COMPARISONS}

\section{Satellite perturbed by Moon and oblate Earth}

For further evaluation of the quality of the methods, a scenario is introduced, in which a satellite about the Earth is perturbed by two forces: the oblateness of the Earth and the gravitation of the Moon. The satellite has a highly elliptical orbit with an eccentricity of approximately $e=0.95$. Thus, it moves very fast at its perigee and in this region it is strongly perturbed by the oblateness of the Earth. At apogee the satellite is subject to perturbations caused by the moon, which are very significant due to its low velocity in this region.

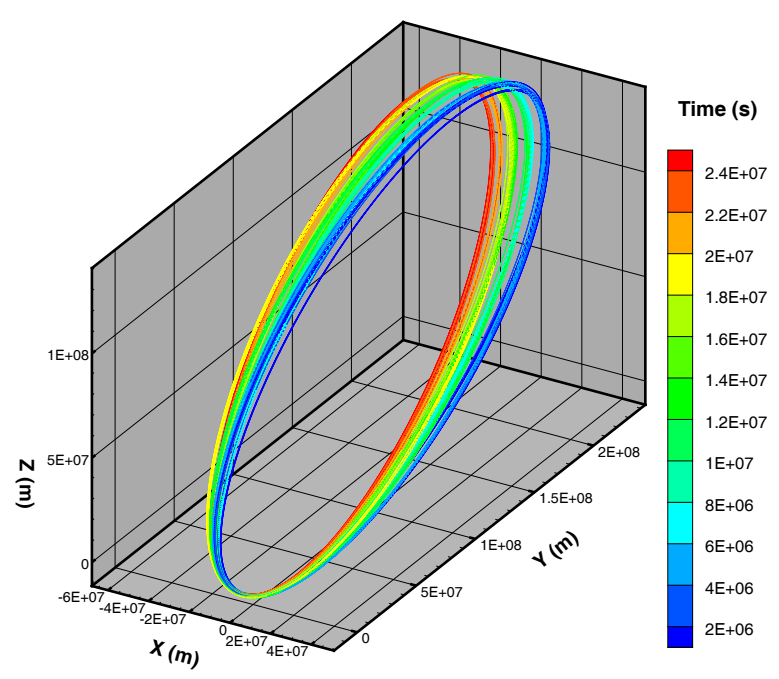

Figure 4. Trajectory of Stiefel \& Scheifele's Example $2 \mathrm{~b}$, extracted from ${ }^{21}$

Problem description The initial conditions of the problem are given as coordinates and velocity components at $t_{0}$. They refer to the cartesian coordinate system associated with the center of the Earth and have the values

$$
\begin{aligned}
& \mathbf{r}_{0}=(0.0,-5888.9727,-3400.0) \mathrm{km} \\
& \mathbf{v}_{0}=(10.691338,0.0,0.0) \mathrm{km}
\end{aligned}
$$

This corresponds to a perigee height of 6800 kilometers. The span of the integration is equal to 288.12768941 days, which roughly describes 50 complete orbits. The perturbation due to the Earth's oblateness, defined by the $J_{2}$-term, the value for the Earth radius $R_{E}$, and the Earth gravity constant $\mu$ are given by

$$
\left.\begin{array}{rl}
J_{2} & =1.08265 \times 10^{-3} \\
R_{E} & =6371.22 \mathrm{~km} \\
\mu & =398601 \mathrm{~km}^{3} \mathrm{~s}^{-1}
\end{array}\right\}
$$

The perturbations due to the moon and the Earth's oblateness are calculated as explained in [21, p. 122]. Hence, the force of the Moon's gravity acting on the satellite is defined through equation

$$
\ddot{\mathbf{r}}=G m_{3}\left(\frac{\mathbf{r}_{3}-\mathbf{r}}{\left\|r_{3}-r\right\|^{3}}-\frac{\mathbf{r}_{3}}{r_{3}^{3}}\right) .
$$

In this equation, $\mathbf{r}_{3}$ is the lunar position vector and $\mathbf{r}$ is the satellite position. The lunar gravity constant takes the value $\mu_{l}=4902.66 \mathrm{~km}^{3} \mathrm{~s}^{-1}$. The lunar position is not defined by an ephemeris model but by trigonometric functions based on the time of the integration. They read

$$
\mathbf{r}_{3}=r_{3}\left(\sin \Omega_{L} t \mathbf{i}_{1}-\frac{\cos \Omega_{L} t}{2}\left[\sqrt{3} \mathbf{j}_{1}+\mathbf{k}_{1}\right]\right) .
$$

The radius of the lunar orbit and the orbital angular velocity are given by

$$
\begin{aligned}
r_{3} & =384400 \mathrm{~km} \\
\Omega_{L} & =2.665315780887 \times 10^{-6} \mathrm{~s}^{-1}
\end{aligned}
$$

Originally this example was intended to show the performance of the regularization method presented in Stiefel \& Scheifele. ${ }^{21}$ It gave exact initial conditions and a precise result for different numbers of steps. It was shown, that for a high number of steps, no further improvement of the result could be achieved. Hence, the final position obtained by Stiefel \& Scheifele can be assumed very accurate and thus serve as reference. Bond 
\& Allman referred to this example to compare further regularization methods, such as Kustaanheimo \& Stiefel or Sperling \& Burdet. ${ }^{22}$ In their work, the different methods were integrated with a stepsize-controlled RungeKutta-Fehlberg algorithm of 4th/5th order (RKF4(5)). To have equal conditions, the integrator's tolerance is tuned for each regularization method in such a way, that it reaches the final position with approximately the same number of integration steps per orbit. In, ${ }^{2}$ the results of the first version of DROMO were added, yielding the values shown in table 1 . In this table all regularization methods use an RKF4(5) and they reach the final position with 62 steps per revolution. In this chapter, the latest DROMO version, reformulated according to, ${ }^{23}$ and the Störmer-Cowell integrator will be compared using the following scenario.

\begin{tabular}{llllll} 
Method & $\begin{array}{l}\text { Stiefel \& } \\
\text { Scheifele }\end{array}$ & $\begin{array}{l}\text { Sperling \& } \\
\text { Burdet }\end{array}$ & $\begin{array}{l}\text { Kustaanheimo } \\
\text { \& Stiefel }\end{array}$ & Cowell & DROMO \\
\hline $\mathrm{x}(\mathrm{km})$ & -24219.050 & -24218.818 & -24219.002 & -24182.152 & -24219.279 \\
$\mathrm{y}(\mathrm{km})$ & 227962.106 & 227961.915 & 227962.429 & 227943.989 & 227962.207 \\
$\mathrm{z}(\mathrm{km})$ & 129753.442 & 129753.343 & 129753.822 & 129744.270 & 129753.492 \\
steps/rev & 500 & 62 & 62 & 240 & 62 \\
Error $(\mathrm{km})$ & - & 0.318 & 0.501 & 42.5 & 0.250 \\
\hline
\end{tabular}

Table 1. Results for Stiefel \& Scheifele's Example 2b, as obtained in ${ }^{2}$

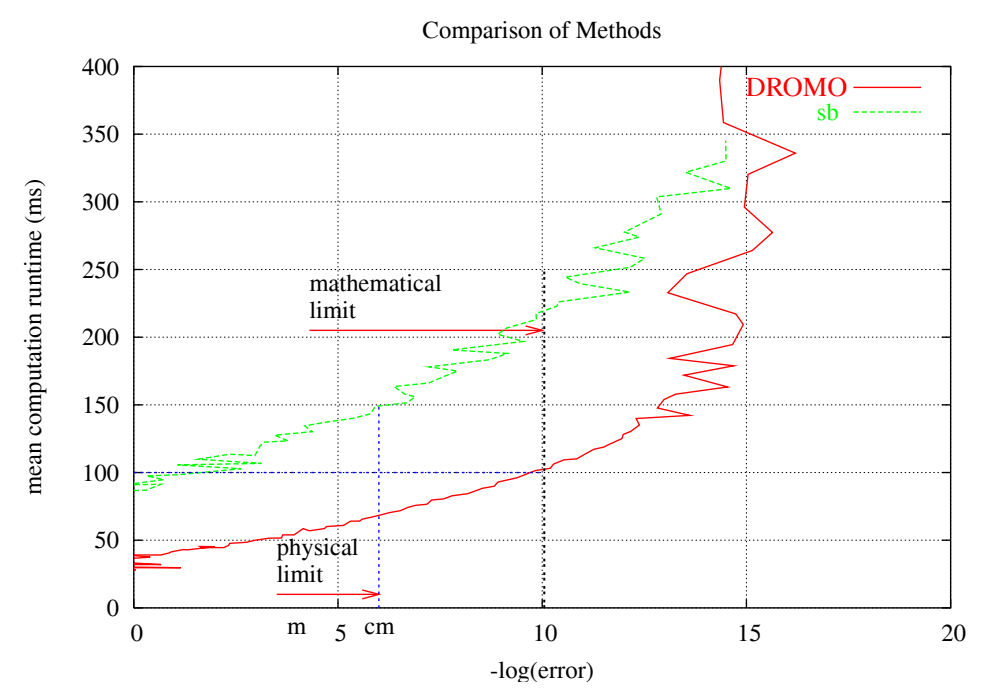

Figure 1. Comparison between DROMO and Sperling-Bürdet

Table 1 shows that DROMO and the Sperling-Bürdet's method provide similar accuracy. An additional comparison between these two methods have been carried out in. ${ }^{2}$ In that comparison the exact solution is not the one given in the book. $^{21}$ Instead, we recalculated the solution two times using both propagators with the maximum accuracy; we took as exact solution the common part obtained in both calculations.

The computations were done: 1) in the same computer (Intel Xeon $3056 \mathrm{MHz}$ microprocessor, 2 Gb RAM), 2) with the same compiler (Intel C++ 8.1.022), 3) with the same integrating algorithm (Runge-Kutta-Fehlberg 7(8) of variable step-size), and 4) in the same computer conditions (processor load, etc). To minimize the effect of uncontrolled factors on the computation time, propagations were repeated 30 times and the mean value of runtime was obtained.

Figure 1 - taken from ${ }^{2}$ - shows the results of the comparison. The runtime is plotted in ordinates and the common logarithm of the norm of the error vector $(-\log (|\Delta \bar{x}|)$ in abscissas. This last quantity is a measure of the quality of the solution: it is approximately equivalent to the number of exact decimal digits of the solution plus one.

The plot shows better performances for DROMO; it seems to be quicker for the same precision, or equivalently, it seems to be more accurate for identical computational time. These differences are mainly due to the lower order of DROMO (8 ODE's) compared with the Sperling-Bürdet's method (13 ODE's). But there are other reasons also: in the Sperling-Bürdet's method the calculation of the "second members" of equations requires to process perturbation forces through numerical treatments of some length; this also happens 
in similar methods based on regularization techniques as the Kustaanheimo-Stiefel method. In our method however, forces hardly require manipulation. Note that the right hand sides of equations only include their components in the orbital frame, which are obtained by simple scalar products. Moreover, the simplicity of programming, joined to the clearness and the simplicity of equations governing the evolution of Euler parameters, strengthens our conviction in the method's advantages.

The comparison performed in table 1 and other similar performed in other contexts ( $\mathrm{see}^{24,25}$ in the field of interplanetary trajectories) is not completely fair for the Cowell method. The reasoning is as follows: for any method, DROMO or Cowell's method, exist a numerical integrator that provides the best performances of the method by achieving the required numerical accuracy after propagation over some specified simulation time. The point is that these integrators need not be the same for the different methods considered. Thus, for each method, we should select the numerical integrator that minimizes the CPU time needed to achieve the specified error.

\section{A finer comparison of propagators}

The results of table 1 arise from a comparison of different propagators, intended to highlight the achievable accuracy when the steps per revolution ratio is held constant* and the integrator used is the same for all cases. This seems adequate for an equitable comparison of propagators when the used integrator is common but the method (i.e. the system of ODE equations to be integrated) is not. However, if we wish to compare propagators using different integrators for each method, then fixing the steps/revolution ratio does not seem fair any more, since multi-step integrators take a single function evaluation per step, whereas single-step integrators make several function calls per step. Thus, a better criteria for fairly comparing propagators seems one based on the computational cost, so, we prefer to compare the achievable accuracy for equal function calls. This is measured by comparing the final error for each propagator when their tolerance is tuned so that the number of function calls remains the same (372 calls), which more equitably quantifies the performance of the propagators. Table 2 and figure 2 gather the main results of our tests.

\begin{tabular}{llllll} 
Method & Cowell & DROMO & DROMO & Cowell & Cowell \\
\hline Integrator & RKF4(5) & RKF4(5) & RKF7(8) & Störmer-Cowell 5 & Störmer-Cowell 9 \\
\hline $\mathrm{x}(\mathrm{km})$ & -24210.188 & -24219.049 & -24219.050 & -24232.184 & -24219.183 \\
$\mathrm{y}(\mathrm{km})$ & 227957.706 & 227962.097 & 227962.105 & 227966.173 & 227962.169 \\
$\mathrm{z}(\mathrm{km})$ & 129751.208 & 129753.437 & 129753.441 & 129755.268 & 129753.473 \\
steps/rev & 240 & 62 & 29 & 372 & 372 \\
Fcalls/rev & 1440 & 372 & 372 & 372 & 372 \\
Run-Time(s) & 0.232 & 0.094 & 0.050 & 0.065 & 0.12 \\
Error $(\mathrm{km})$ & 10.143 & 0.010 & 0.002 & 13.896 & 0.150 \\
\hline
\end{tabular}

Table 2. Results for Stiefel \& Scheifele's Example 2b, using DROMO's newest formulation and different integrators for the Cowell equations.

A glance at table 2 evidences very interesting results. We see that Cowell equations integrated with a Störmer-Cowell integrator of order 5 (SC5), and constrained to 372 function calls per orbit, provide a final error of 13.896 kilometers, which is an accuracy unachievable with a RKF4(5) integrator unless we permit it to make up to 1440 function calls per orbit, which involves a computational cost almost four times larger. This accuracy for the Cowell formulation can though be enhanced by the use of higher order integrators, as allowing the variable order Störmer-Cowell integrator to increase its order up to order 9 (SC9), thus providing a much smaller final error of just 150 meters, but with a slightly increased run-time ${ }^{\dagger}$. However, the best performance in table 2 is shown by the DROMO regularization method, even when integrated with a simple

\footnotetext{
* Notice that the steps per revolution ratio was fixed to 62 for regularization methods, but for the Cowell formulation had to be increased to 240 if comparable final errors were to be obtained.

${ }^{\dagger}$ The increase in run-time is due to the overhead of calculating a larger table of divided differences, which becomes visible when function evaluations are computationally cheap, as is the case
} 
low-order RKF4(5). In this conditions, DROMO yields a final error of only 10 meters, with a run-time that competes with that of SC5, but with an error yet an order of magnitude smaller than that provided by the SC9 integrator. If the integration routine used with DROMO is updated to RKF7(8) the error decreases until $2 \mathrm{~m}$, practically two order of magnitude smaller than that provided by the SC9 integrator.

At this point we must remark that the results for DROMO gathered in table 2, which show an improvement in accuracy compared with the previous results of table 1 taken from, ${ }^{2}$ are mainly due to a slight reformulation of DROMO equations introduced in, ${ }^{23}$ which enhances the the algorithm's performance, and the use of improved Runge-Kutta-Fehlberg routines.

Hence, after observing that in terms of precision the Cowell method cannot compare to DROMO when using an integrator of the same order, the question arises of what could be the increase in performance for DROMO when using high-order integrators instead. For these purpose, we have run several simulations within this scenario with both DROMO and Cowell formulations and different high-order integrators, by sequentially tightening the integration tolerance and plotting their performance in a "Run-Time" versus "Final Error" graphic (figure 2), which gives a clearer insight of the overall performance of these propagators, by directly relating their computational cost to the accuracy the provide.

We easily find out that among the propagators tested the best performance is reached by DROMO and the variable order (up to order 8) Shampine-Gordon integrator, which takes advantage of the higher efficiency of multistep integrators as compared to high-order Runge-Kutta integrators. In fact, it can be observed that for a given run-time DROMO is more accurate, or interpreted the other way around, for a given accuracy DROMO is faster, thus concluding that for the current scenario of a highly perturbed orbital motion, DROMO shows an outstanding performance when integrated with high order integrators, far beyond that obtainable by StörmerCowell propagators.

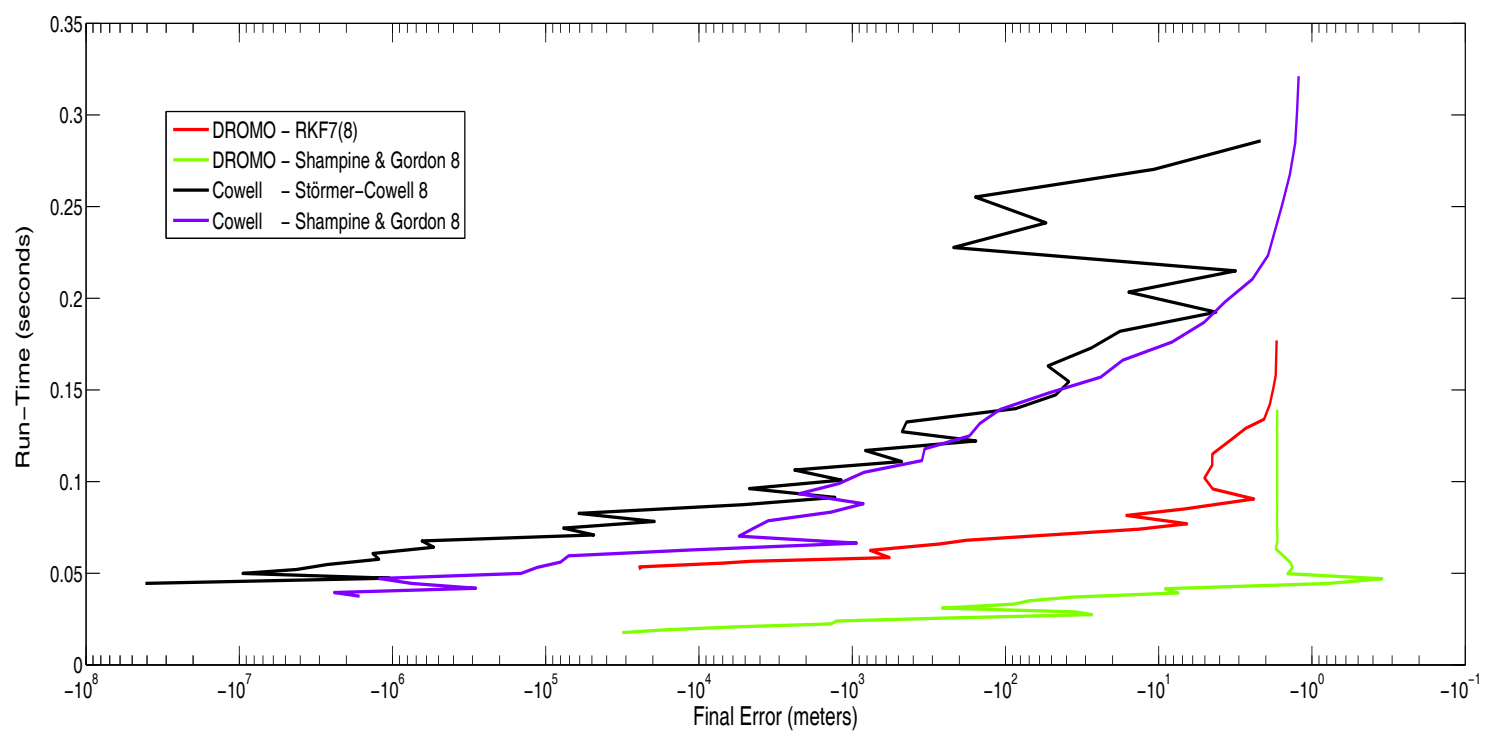

Figure 2. Comparative results showing the "Run-Time" vs "Final Error" relation for different propagators when used in Stiefel \& Scheifele's Example 2 b.

Additional comparatives have been performed in ${ }^{26}$ using the Tsien problem as scenario.

\section{CONCLUSIONS}

From the analysis carried out in this paper some conclusions can be drawn. 
- In terms of accuracy DROMO with the Runge-Kutta-Fehlberg routine RKF7(8) turn out to be the best combination since, in general, they provide a longer and more stable integration and a much more accurate answer (in the example $2 \mathrm{~b}$ of ${ }^{21}$ ).

- In terms of function calls the Störmer-Cowell formulation -in some cases, but not always- turns out to be the best formulation since it provides the lower number of call to the derivative functions.

That is, DROMO + RKF7(8) reaches levels of accuracy unachievable for other propagators. Due to the plus of accuracy provided by the DROMO formulation, this scheme is the most appropriated for the propagation of orbits when a high-fidelity description of the trajectory is mandatory. This plus of accuracy, however, has a cost: the higher number of function calls due to the Runge-Kutta-Fehlberg routine used to perform the integration.

However, and from a global point of view, the combination of DROMO with the multistep method of Shampine \& $\operatorname{Gordon}^{27}$ (DE) shows excellent characteristics because: 1) the accuracy worsens in a small amount, relative to the accuracy provided by the combination DROMO + RKF7(8), and 2) the number of function calls reduce in a significant way (see figure 2). Regarding this last point, it should be noticed that the Störmer-Cowell formulas requires one function call per step, and the multistep method of Shampine \& Gordon ${ }^{27}$ (DE) requires two function call per step due to the second evaluation that takes place in the correction part of the algorithm.

The runtime is not a reliable parameter because: 1) is influenced by the MATLAB environment in which most of the calculations have been made, and 2) the simplicity of the derivatives in the problems used to test the different schemes leads to an almost zero computational cost which cannot be extrapolated to the propagation of real orbits.

In any case, this paper is an approximation that would be duly qualified in the future.

\section{ACKNOWLEDGMENT}

This work was carried out in the framework of the research project entitled Dynamic Simulation of Space Complex Systems (AYA2010-18796) supported by the Spanish Ministry of Economy and Competitiveness. Authors thank Spanish Gov. by its support.

\section{REFERENCES}

[1] J. Peláez and J. M. Hedo, "Un método de Perturbaciones Especiales en Dinámica de Tethers," Monografías de la Real Academia de Ciencias de Zaragoza, Vol. 22, 2003, pp. 119-140.

[2] J. Peláez, J. M. Hedo, and P. R. d. Andrés, "A special perturbation method in orbital dynamics," Celestial Mechanics and Dynamical Astronomy, Vol. 97, 2007, pp. 131-150. 10.1007/s10569-006-9056-3.

[3] A. Deprit, "Ideal elements for perturbed Keplerian motions," Journal of Research of the National Bureau of Standards. Sec. B: Math. Sci., Vol. 79B, No. 1-2, 1975, pp. 1-15. Paper 79B1\&2-416.

[4] A. Deprit, "Ideal frames for perturbed Keplerian motions," Celestial Mechanics and Dynamical Astronomy, Vol. 13, No. 2, 1976, pp. 253-263.

[5] M. Palacios, A. Abad, and A. Elipe, "An efficient numerical method for orbit computations," Astrodynamics 1991, Vol. 1, 1992, pp. 265-274.

[6] M. Palacios and C. Calvo, "Quaternions formulation versus regularization in numerical orbit computation," Astrodynamics 1993, 1993, pp. 2629-2644.

[7] M. Palacios and C. Calvo, "Ideal frames and regularization in numerical orbit computation," Journal of the Astronautical Sciences, Vol. 44, No. 1, 1996, pp. 63-77.

[8] R. Cid and M. San Saturio, "Motion of rigid bodies in a set of redundant variables," Celestial Mechanics and Dynamical Astronomy, Vol. 42, No. 1, 1987, pp. 263-277.

[9] M. Sharaf, M. Awad, and S. Najmuldeen, "The motion of artificial satellites in the set of Eulerian redundant parameters," Earth, Moon, and Planets, Vol. 55, No. 1, 1991, pp. 21-44.

[10] M. Sharaf, M. Awad, and S. Najmuldeen, "Motion of artificial satellites in the set of Eulerian redundant parameters, (II)," Earth, Moon, and Planets, Vol. 55, No. 3, 1991, pp. 223-231.

[11] M. Awad, "Oblateneess and drag effects on the motion of satellites in the set of Eulerian redundant parameters," Earth, Moon, and Planets, Vol. 62, No. 2, 1993, pp. 161-177. 
[12] M. Awad, "Analytical solution to the perturbed $J_{2}$ motion of Artificial Satellite in terms of Euler parameters," Earth, Moon, and Planets, Vol. 69, No. 1, 1995, pp. 1-12.

[13] R. Broucke and H. Lass, "On redundant variables in Lagrangian mechanics, with applications to perturbation theory and KS regularization," Celestial Mechanics and Dynamical Astronomy, Vol. 12, No. 3, 1975, pp. 317-325.

[14] R. Barrio and S. Serrano, "Performance of perturbation methods on orbit prediction," Mathematical and Computer Modelling, Vol. 48, No. 3, 2008, pp. 594-600.

[15] J. Danby, Fundamentals of celestial mechanics, Vol. 1. 1992.

[16] G. Hintz, "Survey of orbit element sets," Journal of guidance, control, and dynamics, Vol. 31, No. 3, 2008, pp. 785-790.

[17] M. Efroimsky and P. Goldreich, "Gauge freedom in the N-body problem of celestial mechanics," Astronomy and Astrophysics, Vol. 415, No. 3, 2004, pp. 1187-1199.

[18] J. Peláez, J. M. Hedo, and P. Rodriguez, "A special perturbation method in orbital Dynamics," AAS/AIAA Spaceflight Mechanics Meeting 2005 (D. A. Vallado, M. J. Gabor, and P. N. Desai, eds.), Vol. 120 of Advances in the Astronautical Sciences, 2005, pp. 1061-1078.

[19] M. Sharaf, M. Awad, and S. Najmuldeen, "Motion of artificial satellites in the set of Eulerian redundant parameters (III)," Earth, Moon, and Planets, Vol. 56, No. 2, 1992, pp. 141-164.

[20] J. L. G. Gómez, "Perturbation Methods In Optimal Control Problems Applied To Low Thrust Space Trajectories," Master's thesis, ETSI Aeronáuticos, Technical University of Madrid (UPM), 2012.

[21] G. S. E. L. Stiefel, "Linear and Regular Celestial Mechanics.," 1971.

[22] V. R. Bond and M. C. Allman, Modern Astrodynamics: Fundamentals and Perturbation Methods. Princeton: Princeton University Press, 1996.

[23] G. Baù, C. Bombardelli, and J. Peláez, "Adaptive Scheme for Accurate Orbit Propagation,” Vol. 15, p. 35131.

[24] J. Esteban-Dones and J. Peláez, "Advanced propagation of interplanetary orbits in the exploration of jovian moons," 4th Int. Conference on Astrodynamics Tools and Techniques, 2010, pp. 3-6.

[25] J. Esteban-Dones and J. Peláez, "Advanced Propagation of Interplanetary Orbits in the Exploration of Jovian Moons," 5th International Workshop and Advanced School "Spaceflight Dynamics and Control”, 2010. 17-19 March 2010, Covilha, Portugal.

[26] G. Baù, A. Huhn, H. Urrutxua, C. Bombardelli, and J. Peláez, "DROMO: A New Regularized Orbit Propagator," International Symposium on Orbit Propagation and Determination, 2011. 26-28, September, 2011, Lille, France.

[27] L. F. Shampine and M. K. Gordon, "Local Error and Variable Order Adams Codes," Applied Mathematics and Computation, Vol. 1, 1975, pp. 47-66. 